Emotion Socialization as a Dynamic Process across Emotion Contexts

\author{
Jessica P. Lougheed ${ }^{1}$, Miriam Brinberg ${ }^{2}$, Nilam Ram ${ }^{2,3}$, \& Tom Hollenstein ${ }^{4}$ \\ ${ }^{1}$ Purdue University \\ ${ }^{2}$ The Pennsylvania State University \\ ${ }^{3}$ German Institute for Economic Research (DIW Berlin) \\ ${ }^{4}$ Queen's University
}

Author Note

Jessica P. Lougheed, Department of Human Development and Family Studies, Purdue University; Miriam Brinberg, Department of Human Development and Family Studies, The Pennsylvania State University; Nilam Ram, Department of Human Development and Family Studies, The Pennsylvania State University; Tom Hollenstein, Department of Psychology, Queen's University.

We gratefully acknowledge the support of a Banting postdoctoral fellowship from the Social Sciences and Humanities Research Council of Canada (Jessica P. Lougheed), the Penn State Biomedical Big Data to Knowledge Predoctoral Training Program funded by the National Library of Medicine (T32 LM012415; Miriam Brinberg), and grant 386479-2011 from the Natural Sciences and Engineering Research Council of Canada (Tom Hollenstein).

Correspondence concerning this article should be addressed to Jessica P. Lougheed, Department of Human Development and Family Studies, Purdue University, 1200 West State Street, West Lafayette, IN, 47907-2055. E-mail: jlougheed@purdue.edu replicate the final, authoritative version of the article. Please do not copy or cite without authors' permission. The final article will be available, upon publication, via its DOI: 10.1037/dev0000801 


\begin{abstract}
Emotion-related socialization behaviors that occur during parent-child interactions are dynamic. According to Eisenberg, Cumberland, and Spinrad's (1998) model, on-going parental reactions to emotions and discussions of emotion indirectly shape children's socioemotional competence throughout childhood and adolescence. Typically-developing adolescents_-girls especially—are at increased risk for developing internalizing symptoms. We examined if and how emotion dynamics of mother-daughter interactions contribute to adolescent girls' internalizing symptoms. We applied grid-sequence analysis (Brinberg et al., 2017) to observational data obtained while $N$ $=96$ typically-developing adolescent girls $\left(M_{\text {age }}=13.99\right.$ years $)$ and their mothers engaged in five different emotionally-laden discussions. We identified patterns of expressed emotions that unfolded during each discussion and examined how interdyad differences in those patterns were associated with mothers' and daughters' internalizing symptoms. Dyads differed with respect to whether mothers or daughters tended to regulate positive emotion expressions. Interdyad differences in moment-to-moment dynamics of happy/excited and worried/sad discussions were associated with adolescent girls' social anxiety symptoms, while differences in emotion dynamics of proud, frustrated/ annoyed, and grateful discussions were not. Taken together, results illustrate how methodological innovations are enabling new examination and detailed description of parent-child emotion socialization dynamics.

Keywords: Emotion socialization; parent-adolescent relationships; internalizing symptoms; sequence analysis
\end{abstract}




\section{Emotion Socialization as a Dynamic Process across Emotion Contexts}

Eisenberg and colleagues (1998) forwarded a model of parental emotion socialization that laid the groundwork for the following two decades of research on the role of parent-child interactions in children's developing socioemotional competence. This model delineated the process of emotion socialization at multiple levels, including the broader emotional context, specific emotion-related parenting practices, and outcomes for child development. The outcomes described in the model, such as child socioemotional competence, in turn feed back into the characteristics that set the stage for specific parenting practices and child emotional responses (Eisenberg, Cumberland, et al., 1998; Eisenberg, Spinrad, \& Cumberland, 1998).

Specific parental emotion-related socialization behaviors (ERSBs) are a central concept in this model. The core of emotion socialization occurs through ERSBs such as parental reactions to children's emotional expressions, discussion of emotions with their children, and emotional expressiveness. Parents can use such behaviors to both directly teach children and indirectly model the appropriate control and expression of emotion according to situational demands (Eisenberg, Cumberland, et al., 1998; Morris, Silk, Steinberg, Myers, \& Robinson, 2007). Children, in turn, internalize how to express and control their emotions and eventually become more independent in their emotion regulation ability. Thus, emotion socialization is a dynamic process that unfolds over the course of parent-child interactions.

The context (emotional valence and tone of a specific situation) of parent-child interaction is also central to Eisenberg et al.'s (1998) model. The emotional context of a parentchild interaction is expected to influence which ERSBs parents show. For example, some parents may be more likely to respond punitively to children's negative emotion expressions in highly stressful contexts compared to contexts that are positively valenced (Eisenberg, Cumberland, et 
al., 1998). In addition, the appropriateness of parental ERSBs in context is expected to influence children's level of emotional arousal. For example, if a parent reacts to a child's expression of sadness with anger, the child's arousal level may increase and lead to amplified experience of negative emotion (e.g., Granic \& Lougheed, 2016). In contrast, if a parent reacts supportively to a child's expression of sadness, the child's arousal level may decrease and eventually downregulate the experience of negative emotion (Lougheed, Hollenstein, Lichtwarck-Aschoff, \& Granic, 2015). Therefore, the emotional context of parent-child interactions is expected to play a role in which parental ERSBs are observed, and in turn, influence children's emotional experience and expression in that context.

Research on emotion socialization in the last twenty years has made significant steps forward in providing evidence for Eisenberg et al.'s (1998) model. However, there remains much to be learned about socialization as a dynamic process. Two primary approaches have been used to examine emotion socialization to date. One approach involves using self-report measures of parental responses to child emotions (e.g., Fabes, Leonard, Kupanoff, \& Martin, 2001). This method is valuable because it yields information about parental tendencies to respond to a range of child emotions. However, this approach is limited in that it relies on participants' insight about their own behaviors, and it cannot provide information about specific instances of parental reactions to children's emotions. The second approach is to conduct behavioral observations of parent-child interactions and code behaviors and emotions (e.g., Hastings, Klimes-Dougan, Kendziora, Brand, \& Zahn-Waxler, 2014). The advantages of this approach are that it does not rely on parental insight, and the emotional context of the interaction can be accounted for. However, such data are often either coded for dynamics and aggregated across time into summary frequency counts or total durations, or coded via global ratings of the entire interaction. 
Both approaches obscure the process of socialization as it unfolds moment-to-moment during the interaction. It is crucial to examine the process of socialization as it unfolds during interactions to better understand its associations with child outcomes and socioemotional competence (Morris et al., 2007). In the current study, we use a novel approach that captures the sequence of motherdaughter emotion expressions to examine socialization dynamics across different emotion contexts in adolescence.

\section{Emotion Socialization in Parent-Adolescent Dyads}

The socialization of emotion changes according to child development. It is considered to be a parent-driven process in infancy and early childhood, with parental ERSBs exerting greater influence over child emotions than the other way around (Eisenberg, Cumberland, et al., 1998). The process becomes increasingly bi-directional as children get older. Children and preadolescents become increasingly able to effect change in their parent's emotions and behaviors during interactions as they become more autonomous (Eisenberg, Cumberland, et al., 1998; Klimes-Dougan et al., 2007; Morris et al., 2007). By adolescence, the parent-child relationship begins to shift from one that is more hierarchical to one that is more egalitarian (Collins \& Laursen, 2004; Smetana, Campionne-Barr, \& Metzger, 2006). The transforming parent-adolescent relationship can be seen in age differences in the emotion dynamics of parentadolescent interactions. For example, older adolescents tend to supportively validate maternal emotion expressions more than younger adolescents (Main, Paxton, \& Dale, 2016). By validating maternal emotions, older adolescents may be facilitating their mothers' emotion regulation and communicating that the expression of those emotions is acceptable (i.e., emotion socialization).

Emotion socialization in adolescence is associated with adolescent psychosocial adjustment (e.g., Morris, Criss, Silk, \& Houltberg, 2017). Adolescents experience increased risk 
for psychosocial adjustment difficulties such as internalizing symptoms (e.g., depression, anxiety; Dahl, 2001). Age-typical emotional developments such as increased emotional intensity and lability, and related difficulties with emotion regulation, are one process underlying this increased risk (Dahl, 2001). Parental emotion socialization behaviors during this transitional period can protect adolescents from increased risk or exacerbate psychosocial adjustment difficulties, depending on whether parental behaviors support or undermine the development of adolescent emotion regulation. For example, parents of adolescents experiencing depression tend to report responding to their adolescents' expressions of positive emotions in ways that dampen or minimize their positive feelings (Katz et al., 2014). Depressive symptoms are associated with difficulties experiencing and up-regulating positive emotions (Fussner, Luebbe, \& Bell, 2014), and parents who dampen their adolescents' positive expressions may be communicating that those emotions are unacceptable. There is also evidence that punitive maternal responses to adolescent negative emotions may increase the risk for adolescent depressive symptoms longitudinally, whereas supportive maternal responses may decrease the risk (Hastings et al., 2014).

A small number of studies have "zoomed in" on the dynamics of unfolding parentadolescent interactions to examine the process of emotion socialization. Such studies enable researchers to examine dynamics related to socialization processes such as emotional and behavioral synchrony (Morris, Cui, Criss, \& Simmons, 2018; Pitzer \& Bergeman, 2014). Whether interaction partners respond to each other in a way that is "in tune" with each other's behaviors (synchrony) or not (asynchrony) is likely related to how emotions are socialized in the family (Eisenberg, Cumberland, et al., 1998). Mothers may be more likely than adolescents to respond to negative expressions with positive emotions, whereas adolescents may be more likely 
to reciprocate and thereby escalate negative expressions (van Bommel, van der Giessen, van der Graaff, Meeus, \& Branje, in press). However, when mothers do reciprocate adolescent girls’ aggressive expressions, adolescents may be at increased risk for developing major depressive disorder in the future (Schwartz et al., 2011). There is also evidence that mothers may play an important role in resolving cycles of reciprocated emotions, and that maternal resolution of such cycles is associated with adolescent psychosocial adjustment. When such cycles are not resolved by mothers, there tends to be an increased risk of adolescent behavioral problems (Moed et al., 2015). In addition, initial evidence suggests that older adolescents tend to drive negative emotion cycles whereas mothers tend to drive them with younger adolescents (Main et al., 2016). In terms of supportive maternal behaviors, mothers are less likely to supportively regulate their adolescents during interactions for adolescents higher in depressive symptoms (Lougheed, Craig, et al., 2016), but may be more likely to help their daughters down-regulate negative emotions if they show greater developmental risk (i.e., early pubertal maturation; Lougheed, Hollenstein, \& Lewis, 2016).

These studies of socialization dynamics have used methods that involve specifying types of emotional exchanges during parent-adolescent interactions (e.g., negative emotion synchrony and reciprocity, specific maternal responses to emotions), such as sequential analysis (Schwartz et al., 2011; van Bommel et al., in press), survival analysis (Lougheed, Craig, et al., 2016; Lougheed, Hollenstein, et al., 2016), recurrence quantification analysis (Main et al., 2016), and by calculating variables indicating episodes of reciprocated negative emotion exchanges for use in other analysis types (Moed et al., 2015). These studies give insights into understanding socialization as a dynamic process, especially in terms of specific emotional exchanges of interest. In line with Eisenberg et al.'s model (1998), it is also important to consider the emotion 
dynamics of the entire interaction, in addition to specific patterns of sequences and contingencies between parents' and adolescent's emotional expressions. Interpersonal interactions are an unfolding process consisting of complex patterns of emotional expressions and responses. To date, studies taking a dynamic approach have focused on single-step sequences rather than complex multi-step sequences that show patterns of emotion socialization across the history of an interaction.

\section{The Current Study}

In the current study, we build on previous work by capturing multi-step sequences of interpersonal emotion expressions during parent-adolescent interactions. These sequences are the temporally-ordered emotions expressed moment-by-moment during interactions, such as the following three-step sequence in which the members of a dyad begin an interaction (1) expressing neutral emotion; then (2) the daughter makes a joke and laughs, expressing a positive emotion; which is then (3) reciprocated by the mother's laughter. We examined such sequences for the entire duration of five different positively- and negatively-valenced discussions of specific emotional experiences in the parent-adolescent relationship. This approach allowed us to describe socialization dynamics in different contexts in which emotional experiences are discussed, in line with Eisenberg et al.'s (1998) assertion that the discussion of emotional experiences is one avenue of emotion socialization. Specifically, we examined dynamics in five different emotion contexts: (1) Happy/Excited, (2) Worried/Sad, (3) Proud, (4)

Frustrated/Annoyed, and (5) Grateful. These five different contexts represent a range of positive and negative emotions that differ in their functional significance for interpersonal interactions (Walle \& Campos, 2012). For example, the discussion of sadness experiences may elicit greater interpersonal connectedness as partners attempt to alleviate the other's emotions, whereas the 
discussion of anger experiences may incite more synchronous negative expressions as partners assert their opposing views. Moreover, discussing positive topics (Happy/Excited) may elicit the mutual up-regulation of positive emotion states (positive synchrony; Ramsey \& Gentzler, 2015), whereas discussing prideful and grateful emotions may elicit a mix of positive and negative emotions (e.g., sadness in the case of nostalgia or for overcoming a negative experience).

Grid-sequence analysis (Brinberg, Fosco, \& Ram, 2017; Brinberg, Ram, Hülür, Brick, \& Gerstorf, 2018) is a novel approach merging state space grid methods from developmental psychology (Hollenstein, 2013) with sequence analysis from biology (Kruskal, 1983). State space grids are a dynamic systems approach to analyzing how individuals or dyads change over time. Sequence analysis has been commonly used to identify and group biological sequences such as DNA. We used grid-sequence analysis to visualize, identify, and quantify patterns of mother-daughter emotion expressions during the five different emotion contexts. We then examined if and how those patterns were related to mothers' and daughters' internalizing symptoms (e.g., depression, general anxiety, and social anxiety). Mothers and their adolescent daughters were the focus of the current study as adolescent girls experienced increased risk for internalizing symptoms compared to adolescent boys (Graber, Sontag, Lerner, \& Steinberg, 2004), and females tend to be more emotionally expressive than males (e.g., Brebner, 2003).

Our first research question was: Are there interdyad differences in patterns of motherdaughter emotion expressions in each interpersonal emotion context? We used grid-sequence analysis to identify multi-step sequences of dyadic emotion expressions that distinguished between dyads in the sample. Grid-sequence analysis can be used in a data-driven way to identify patterns from entire interaction sequences (i.e., the entire series of dyadic emotion expressions in one interaction context). This research question was exploratory as no research to 
date has examined emotion sequences greater than two turns in mother-adolescent interactions. However, given past research on negative emotion synchrony in parent-adolescent interactions (Main et al., 2016; Moed et al., 2015), we expected that some dyads would be more likely than others to show patterns of negative emotion synchrony in negative interaction contexts (Worried/Sad and Frustrated/Annoyed), such as one individual expressing a negative emotion, which is then met with a negative expression by the other, before the negative emotions either resolve or continue to escalate.

Our second research question was: Are interdyad differences in emotion patterns in different emotion contexts associated with mothers' and daughters' psychosocial adjustment (symptoms of depression, general anxiety, and social anxiety)? For example, there could be interdyad differences in patterns of negative synchrony: some dyads may be more likely to show such patterns in negative interaction contexts than others, and these interdyad differences may be meaningfully related to psychosocial adjustment. Behaviors involved in emotion socialization can be evaluated in terms of whether they foster or undermine children's and adolescents' psychosocial adjustment (Eisenberg, Cumberland, et al., 1998). Moreover, it is not just adolescents' psychosocial adjustment that is likely to play a role in emotion socialization, as parental adjustment is also associated with emotion dynamics in parent-adolescent dyads (Amole, Cyranowski, Wright, \& Swartz, 2017; Lougheed \& Hollenstein, 2016; Morgan et al., 2015). We built upon the data-driven grid-sequence analysis conducted for our first research question to identify interdyad differences in dyadic patterns and did not have specific hypotheses regarding the multi-step patterns that would be identified in the data. However, based on previous research, we anticipated that if we observed interdyad differences as described above, that patterns of negative synchrony would be positively associated with both mothers' and 
daughters' internalizing symptoms.

The current study is the first application of grid-sequence analysis to observed (versus self-reported) dyadic emotion expressions. In testing our second research question, we examined if patterns identified by grid-sequence analysis provided additional information beyond what is explained by a commonly used measure of sequence dynamics coming from state space grid methods: sequence entropy (Hollenstein, 2013). Entropy is a general, sequence-level quantification of the "unpredictability" of a parent-adolescent dyad's emotion expressions (e.g., van der Giessen, Branje, Frijns, \& Meeus, 2013). Low entropy scores indicate low variability in how a dyad's discussion progresses - a low number of transitions among only a few states. In contrast, high entropy scores indicate high variability (or high unpredictability) in how a dyad's discussion progresses - a high number of transitions among a wider range of dyadic emotion expressions (Hollenstein, 2013; Koffer, Ram, \& Almeida, 2017). Thus, our analyses specifically examine if patterns of mother-daughter emotion expressions provide additional information about mothers' and daughters' psychosocial adjustment, beyond differences in overall variability of their emotion expressions.

\section{Method}

\section{Participants}

Participants were an extant sample of 96 typically-developing adolescent females and their mothers (see Lougheed \& Hollenstein, 2016 for complete demographic information). Adolescents were between 13 and 16 years old $(M=13.99, S D=0.87)$, and mothers were between 32 to 57 years old $(M=44.68, S D=5.40)$. Approximately $50 \%$ of mothers reported their gross family income as greater than $\$ 100,000$ per year, and $77 \%$ reported their marital status as married. The majority of mothers identified their own (82\%) and their daughter's (77\%) 
ethnicities as European Canadian. Mothers and daughters each received \$15 for their participation. All procedures for the study (Lougheed \& Hollenstein, 2016) were approved by the Queen’s University Health Sciences Research Ethics Board (File \#6004467).

\section{Procedure}

Mothers and daughters visited a university-based observational laboratory equipped with obscured video cameras and psychophysiological recording equipment that were monitored from an adjacent room (see Lougheed \& Hollenstein, 2016 for full details). Mothers and daughters were seated in comfortable chairs beside each other. Mothers provided informed consent for their own and their daughter's participation, and daughters provided assent, after reviewing a letter of information. A female experimenter then introduced participants to the general procedures of the study. First, participants filled out a series of web-based questionnaires that included demographics and assessments of psychosocial adjustment (e.g., internalizing symptoms).

Next, female experimenters applied physiological sensors to participants. Sensors measured electrodermal activity, heart rate, and respiration, but these physiological data were not analyzed as part of the current study. Participants' normal resting physiological states were measured while participants sat quietly watching a neutral nature film clip (2.5 minutes). Following this resting phase, participants separately filled out a questionnaire to determine topics for the five discussions of the Emotional Rollercoaster task (Lougheed \& Hollenstein, 2016), a series of five discussions about the emotions they felt towards each other. The questionnaire asked participants to list one or two possible topics for the following questions: (1) "I felt happy or excited about my mother/daughter when..." (e.g., happy about spending time together); (2) "I felt worried or sad about my mother/daughter when..." (e.g., worried about her well-being); (3) "I felt proud of my mother/daughter when..." (e.g., proud of her accomplishments); (4) "I felt 
frustrated or annoyed towards my mother/daughter when...” (e.g., annoyed at communication difficulties); and (5) "I felt grateful for or positive towards my mother/daughter when..." (e.g., when she does something nice for me). Then, participants completed the Emotional Rollercoaster task. Dyads discussed each of the five discussion topics in a fixed order: (1) Happy/Excited, (2) Worried/Sad, (3) Proud, (4) Frustrated/Annoyed, and (5) Grateful. Each discussion lasted for three minutes and the experimenter prompted dyads when it was time to move on to the next topic. At the end of the lab session, all participants were debriefed and received compensation.

\section{Measures}

Observed emotions. A team of four undergraduate research assistants used the video recordings to code the observable emotions of each participant in each of the five discussions with a five-code version of the Specific Affect Code (Gottman, McCoy, Coan, \& Collier, 1995) developed for this study (SPAFF5; Lougheed \& Hollenstein, 2016). SPAFF5 codes are categorical and capture emotional tone and are based on facial expressions, body language, and verbal characteristics. The five mutually exclusive SPAFF5 codes were an aggregation of original codes and consisted of the following categories: (1) Positive Emotions (humor, joy, and affection); (2) Interest; (3) Neutral; (4) Internalizing Negative Emotions (e.g., fear, sadness); and (5) Externalizing Negative Emotions (e.g., contempt, anger). Coders indicated the onset and offset times using Noldus Observer 11.0 to continuously capture which of the five mutuallyexclusive emotion codes were observed in mothers' and daughters' expressions at any given moment. Coders showed good inter-rater reliability, with the average percent agreement for frequency-sequence based analyses being $77 \%$ and $\mathrm{K}=.72$, and an average duration-sequencebased percent agreement of $99 \%$. The categorical time series of mothers' and daughters' observed emotions was used to create state space grids (visualizations) and sequences (univariate 
time series) for use in analyses (see Data Analysis section for more details). Less than 1\% of data on observed emotions for the full sample across discussions were missing for mothers and daughters.

Entropy. The overall predictability of each individuals' sequence was quantified using Shannon's (1948) entropy. Specifically,

$$
h\left(p_{1}, \ldots, p_{s}\right)=-\sum_{i=1}^{s} p_{i} \log \left(p_{i}\right)
$$

where $s$ is the number of unique states and $p_{i}$ is the proportion of the occurrences of the $i$ th state in a given sequence (Gabadinho, Ritschard, Müller, \& Studer, 2011). Entropy is zero if all states in a sequence are perfectly predictable from the first state (i.e., all states are the same or alternate between two states). Higher entropy values indicate less predictability in terms of the amount of information required to reproduce a sequence (Hollenstein, 2013). We calculated sequence entropy using the seqient function of the TraMineR package in R (Gabadinho et al., 2011).

Depressive symptoms. Mothers' and daughters' severity of depressive symptoms was measured using the 21-item Beck depression inventory, second edition (BDI-II; Beck, Steer, \& Brown, 1996). For each item, participants selected one of four statements that reflected the extent they experienced symptoms in the last two weeks (e.g., $0=I$ do not feel sad; $1=$ Ifeel sad much of the time; 2 = I am sad all the time; and 3 = I am so sad or unhappy I can't stand it). Two items regarding sex and suicidal thoughts were omitted from the daughters' form to comply with the institutional research ethics board. The item pertaining to sex was retained on the mothers' form. This measure showed high internal consistency for mothers $(\alpha=.89)$ and daughters $(\alpha$ $=.96$ ). Level of depressive symptoms was calculated, separately for mothers and daughters, as the mean of all completed items.

General anxiety symptoms. Presence and severity of mothers' and daughters' general 
anxiety symptoms was measured using the 21-item Beck anxiety inventory (BAI; Beck, Epstein, Brown, \& Steer, 1988). For each item, participants selected one of four statements $(0=$ not at all; 3 = Severely, I could barely stand it) that reflected the extent of their symptoms (e.g., "Unable to relax", "nervous") experienced in the last two weeks. This measure showed high internal consistency for mothers $(\alpha=.90)$ and daughters $(\alpha=.91)$. Level of anxiety symptoms was calculated, separately for mothers and daughters, as the mean of all completed items.

Social anxiety symptoms. Daughters' social anxiety symptoms (fear of negative evaluation by others, social avoidance, and distress) were measured with the 13 -item social anxiety scale for adolescents, short form (SAS-A; Myers, Stein, \& Aarons, 2002). Daughters indicated how well each statement characterized themselves (e.g., "I'm afraid that others will not like me") on a 5-point scale $(1=$ not at all; $5=$ all the time $)$. This measure showed high internal consistency $(\alpha=.93)$. Daughters' level of social anxiety was calculated as the mean of all completed items.

Mothers' social anxiety symptoms were measured with the 24-item Liebowitz social anxiety scale (LSAS; Liebowitz, 1987). Mothers used a 4-point scale to indicate how much fear they experience during $(0=$ none; $3=$ severe $)$ and how frequently $(0=$ never; $3=$ usually $)$ they avoid situations (e.g., "Going to a party", "Being the center of attention"). This measure showed high internal consistency $(\alpha=.97)$. Mothers' level of social anxiety was calculated as the mean of all completed items.

\section{Data Analysis}

Grid-sequence analysis was conducted in a series of five steps (Brinberg et al., 2017, 2018). In Stage I, dyad-level categorical time series were created from the raw data. In Stage II, interdyad differences in sequences were examined in relation to our two research questions. 
Stage I: Intra-family analyses. The first stage of grid-sequence analysis involves creating a dyad-level categorical time series by visualizing and quantifying dyadic sequences in grids (Brinberg et al., 2017, 2018). In brief, a state space grid is established for each dyad with each member represented on one axis, then grid cells are labeled, and these labels are used to extract a dyadic categorical time series.

Step 1 (Gridding). The first step is to map the trajectory of mother-daughter observed emotion states for each discussion in a two-dimensional state space. We created a state space grid with mothers' observed emotions on the $x$-axis and daughters' observed emotions on the $y$-axis using the base, ggplot2, and reshape packages in R (R Core Team, 2018; Wickham, 2007, 2009). As shown in Figure 1, panel A, the grids contain 25 cells representing all possible combinations of mothers' and daughters' SPAFF5-based emotion states. Plot points on the grids indicate the duration of time spent in each cell (longer durations indicated by larger plot points), and the trajectory of dyadic states is represented by the lines connecting the plot points (Hollenstein, 2013).

Step 2 (Obtain sequences). Next, we labelled each cell of the grid with letters (see Figure 1, panel A). Then, we extracted each dyads' sequence of letters from the grids in the temporal order that the cells were visited to obtain a dyad-level categorical time series (see Figure 1, panel B) - a "sequence" of the time-ordered dyadic emotion expressions (i.e., lettered grid cells) for each discussion. The sequences were then represented using a wide-format data frame for each discussion, with one row per dyad and 1800 time points (deciseconds) within each discussion. The time unit of deciseconds was selected to map onto the temporal accuracy of the event-based coding scheme (see details in Method). These data frames are depicted visually in Figure 2, with each row representing one dyad's sequence and the colors, moving left to right, indicating the 
order of cells visited during the discussion. Missing values are denoted by light gray.

Stage II: Interdyad differences in mother-daughter dynamics. The second stage of grid-sequence analysis involves examining interdyad differences in sequences. Our first research question was: Are there interdyad differences in patterns of mother-daughter emotion expressions in each interpersonal emotion context? For each of the five discussions, we calculated dissimilarities among all pairs of sequences (see Step 3 below). Then, we used these dissimilarities to examine interdyad differences using multidimensional scaling (MDS; Hout, Papesh, \& Goldinger, 2013). Our second research question was: Are interdyad differences in emotion patterns in different emotion contexts associated with mothers' and daughters' psychosocial adjustment? To answer this question, we examined associations between interdyad differences in sequences and psychosocial adjustment using linear regressions.

Step 3 (Sequence analysis). Before conducting MDS we needed to calculate the dissimilarity (i.e., "distance”) between all possible pairs of sequences in each discussion. We did so using the length of the longest common subsequence (LCS), which quantifies the longest subsequence common among each set of sequence pairs (Studer \& Ritschard, 2016). LCS is an appropriate distance metric to use when researchers are interested in making comparisons based on the order of states but not their temporal position in a sequence. Figure 1 shows example sequence portions for different dyads. As can be seen in Figure 1 (Panel C), Sequence 2 and Sequence 3 both contain the subsequence M-O-M, but in different temporal locations within the respective sequences. The length of the longest common subsequence between Sequence 2 and Sequence 3 is three (M-O-M), just as the length of the longest common subsequence between Sequence 1 and Sequence 2 (C-E-C) is three. This process is extended to compare each dyad's entire sequence with every other dyad's sequence in the same kind of discussion. Thus, we 
obtained five $96 \times 96$ dissimilarity matrices for mother-daughter emotions, one for each discussion, using the TraMineR and TraMineRextras packages in R (Gabadinho et al., 2011; Studer \& Ritschard, 2016).

Step 4 (Exploration of interdyad differences). Interdyad differences in each set of sequences were quantified using MDS, a general data reduction technique (Hout et al., 2013) used here to identify the primary dimensions along which sequences differ (for example, see Ram, Benson, Brick, Conroy, \& Pincus, 2017). For each discussion, we fit a series of MDS models with 1 to 10 dimensions, extracted and plotted measures of "stress," which indicates relative misfit between the estimated distance matrix and the observed distance matrix. Formally, stress is calculated for a given number of dimensions (1 to 10$)$ as

$$
\text { stress }=\sqrt{\frac{\sum\left(d_{i j}-\widehat{d_{l \jmath}}\right)^{2}}{\sum d_{i j}^{2}}}
$$

where $d_{i j}$ and $\widehat{d_{\imath j}}$ are the observed and estimated distances between sequence $i$ ( 1 to $N$ ) and sequence $j$ ( 1 to $N$; de Leeuw \& Mair, 2009; Kruskal, 1964). In the same way that scree plots of the eigenvalues are used to select the number of factors in factor analysis, scree plots of stress values are used to select the number of dimensions in MDS analysis. Implementation of MDS was done separately for each discussion using the smacof package in R (de Leeuw \& Mair, 2009).

For each of the five discussions, interdyad differences were succinctly represented by two underlying dimensions. MDS assigns each sequence a score on the underlying identified dimensions. In this case, each sequence received two scores: one score for the first dimension and another score for the second dimension. The sequences can be plotted in order of their MDS score on either dimension and further examined to identify distinguishing characteristics between the sequences at low and high ends of the dimensions. To characterize the interdyad differences 
captured by each dimension, we identified and confirmed (using Pearson chi square test of independence) the subsequences (i.e., multistep patterns) that best discriminated the dyadic emotion patterns at the highest and lowest quartiles of each dimension (using the seqecmpgroup function of the TraMineR package; Ritschard, Bürgin, \& Studer, 2013). These subsequences were then used to describe and label the ways in which the mother-daughter sequences differed.

Step 5 (Associations between interdyad differences and psychosocial adjustment). We used multiple linear regressions to examine if and how the labeled interdyad differences in sequences were associated with mothers' and daughters' psychosocial adjustment. For each discussion, we conducted six regressions to examine separately how each aspect of psychosocial adjustment — mothers' symptoms of (1) depression, (2) general anxiety, and (3) social anxiety; and daughters' symptoms of (4) depression, (5) general anxiety, and (6) social anxiety-was related to the two dimensions of interdyad differences identified and labeled in Step 4, controlling for differences in sequence entropy.

\section{Results}

\section{Research Question 1: Interdyad Differences in Patterns of Mother-Daughter Emotion Expressions}

Results from the grid-sequence analysis, calculation of sequence distances, and identification of the subsequences that described the two primary dimensions of interdyad differences are shown in Table 1. Specifically, Table 1 indicates the types of transitions that characterized sequence differences for each discussion.

For Discussion 1 (Happy/Excited), the highest quartile of the MDS scores of Dimension 1 was characterized by transitions between mutual (i.e., synchronous) positive (E) and daughter positive/mother neutral (C) before leading into transitions between daughter positive/mother 
neutral (C) and mutual neutral (M). We described this dimension as "mother regulating mutual positive to daughter regulating positive" to capture the two distinct phases of the most discriminating subsequence. For Dimension 2, the highest quartile of MDS scores was characterized by repeated transitions between mutual neutral (M) and mother positive/daughter neutral $(\mathrm{O})$. We described this dimension as "mother regulating positive" because the pattern is characterized by mothers up- and down-regulating their own positive expressions while their daughter maintained neutral expressions. Together, these two dimensions suggest that when discussing feelings of happiness and excitement towards each other, some dyads show a tendency for both mother and daughter to contribute to the regulation of positive expressions, whereas in other dyads, only the mother regulates positive expressions and the daughter does not reciprocate.

For Discussion 2 (Worried/Sad), the highest quartile of MDS scores of Dimension 1 was characterized by a single transition from mutual (synchronous) positive (E) to mother positive/daughter neutral $(\mathrm{O})$, so we described this dimension as "daughter down-regulating mutual positive." The highest quartile of MDS scores of Dimension 2 was characterized by repeated transitions between daughter positive/mother neutral $(\mathrm{C})$ and mutual neutral $(\mathrm{M})$, so we described this dimension as "daughter regulating positive" to capture daughters" up- and downregulation of their positive expressions while their mothers maintained neutral expressions. Together, these two dimensions suggest that when discussing feelings of worry and sadness towards each other, some dyads show patterns characterized by daughters ending mutual positive expressions, whereas daughters in other dyads repeatedly express positive emotions that are not reciprocated by their mothers.

For Discussion 3 (Proud), the highest quartile of MDS scores of Dimension 1 was 
characterized by daughter-led (C) transitions to mutual (synchronous) positive emotions (E) after a period of daughters' transitions between mutual neutral (M) and daughter positive/mother neutral (C). Thus, we described this dimension as "daughter regulating positive to daughter upregulating mutual positive.” Dimension 2 was characterized by repeated transitions between mutual positive (E) and daughter positive/mother neutral (C). We described this dimension as "mother regulating mutual positive" to capture that daughters maintained positive expressions while their mothers transitioned between neutral and positive expressions. Together, these two dimensions suggest that when discussing feelings of pride towards each other, daughters in some dyads tend to take the lead in up-regulating synchronous dyadic positive emotions, whereas mothers in other dyads take the lead in regulating synchronous positive expressions.

For Discussion 4 (Frustrated/Annoyed), the lowest quartile of MDS scores of Dimension 1 was characterized by mother-led $(\mathrm{O})$ transitions to mutual (synchronous) positive emotions (E) from mutual neutral (M), and so we described this dimension as "mother up-regulating mutual positive." For Dimension 2, the lowest quartile was characterized by daughter-led (C) transitions to mutual (synchronous) positive (E) from mutual neutral (M) before shifting to repeated transitions between daughter positive/mother neutral $(\mathrm{C})$ and mutual neutral $(\mathrm{M})$. To capture these two distinct phases of this subsequence, we described this dimension as "daughter upregulating mutual positive to daughter regulating positive." Together, these two dimensions suggest that when discussing feelings of frustration and annoyance towards one another, mothers in some dyads tend to take the lead in synchronous positive emotions, whereas in other dyads, daughters tend more to lead the regulation of synchronous positive emotions.

For Discussion 5 (Grateful), the highest quartile of MDS scores of Dimension 1 was characterized by mother-led $(\mathrm{O})$ transitions to mutual (synchronous) positive $(\mathrm{E})$ from mutual 
neutral (M), before transitioning to another pattern in which daughters maintained neutral expressions while mothers transitioned between positive $(\mathrm{O})$ and neutral $(\mathrm{M})$. We described this dimension as "mother up-regulating mutual positive to mother regulating positive." The highest quartile of MDS scores of Dimension 2 was characterized by repeated transitions between mutual neutral (M) and daughter positive/mother neutral (C), and to capture daughters' up- and down-regulation of their own positive expressions while their mothers maintained neutral expressions, we described this dimension as "daughter regulating positive." Together, these two dimensions suggest that when discussing feelings of gratitude towards each other, mothers in some dyads tend to take the lead in regulating synchronous positive emotion, whereas daughters in other dyads tend to be regulating their own positive emotions that are not reciprocated by their mothers.

\section{Research Question 2: Associations between Interdyad Differences and Psychosocial}

\section{Adjustment}

We then examined whether the interdyad differences captured by the two primary dimensions in the MDS of the dyadic emotion sequences were associated with mothers' and daughters' psychosocial adjustment, controlling for differences in sequence entropy. Results of these regression analyses are shown in Tables 2 and 3. For Discussion 1 (Happy/Excited), the predictors explained $8 \%$ of the differences in daughters' social anxiety symptoms, $F(3,91)=$ 2.55, $p=0.06$ (see Table 2). The dimension characterized by mothers regulating their own positive emotions (Dimension 2) was significantly associated with daughters' level of social anxiety symptoms, $\beta=0.23, p=0.01$, and all other predictors were not significant. Specifically, higher daughter social anxiety symptoms were associated with daughters maintaining neutral expressions while their mothers up- and down-regulated their own positive expressions. All other 
regressions for Discussion 1 were not significant (see Table 2).

For Discussion 2 (Worried/Sad), the predictors explained 9\% of the differences in daughters' social anxiety symptoms, $F(3,91)=3.04, p=0.03$ (see Table 2 ). The dimension characterized by daughters regulating their own positive (Dimension 2) was significantly associated with lower anxiety, $\beta=-0.29, p=0.01$, and all other predictors were not significant. Thus, lower daughter social anxiety symptoms were associated with mothers maintaining neutral expressions while daughters up- and down-regulated their own positive expressions. All other regressions for Discussion 2 (Worried/Sad) were not significant (see Table 2).

Interdyad differences in the two dimensions describing sequences for Discussion 3 (Proud), Discussion 4 (Frustrated/Annoyed), and Discussion 5 (Grateful), were not significantly related to differences in mothers' or daughters' psychosocial adjustment (see Table 3).

In summary, we found interdyad differences in patterns of mother-daughter emotion expressions in each of the five discussions (Research Question 1). In general terms, interdyad differences reflected who- mother or daughter - tended to lead the regulation of positive expressions during discussions and whether or not positive expressions were synchronous. We also found some evidence to suggest that in typically-developing dyads, these interdyad differences may be associated with adolescent social anxiety symptoms, especially in emotion contexts in which dyads are discussing happy/excited and worried/sad emotional experiences (Research Question 2).

\section{Discussion}

Following Eisenberg et al.'s model (Eisenberg, Cumberland, et al., 1998; Eisenberg, Spinrad, et al., 1998), we considered emotion socialization to be a bidirectional process between mothers and their adolescent daughters and examined ERSBs in terms of the patterns of 
emotions exchanged during emotionally-charged discussions. We examined patterns of dyadic emotion expressions in mother-daughter interactions in five different emotion contexts. We identified interdyad differences in the patterns of emotional exchange in each context. Considering all the discussions together, interdyad differences generally pertained to whether mothers or daughters tended to regulate positive emotions, and whether or not positive emotions were synchronous. We also found some interdyad differences were associated with daughters' social anxiety symptoms. We contrast these findings to what we might have found had we used common approaches to examining observations of emotion socialization. Had we aggregated our emotion codes across time into frequency counts or durations of observed emotions, we would have been restricted to analytical approaches such as correlations between observed mother and daughter emotions in each discussion, or regressions examining how internalizing symptoms are related to frequencies or durations of emotions. These approaches would have provided us with information about the strength of association between overall levels of mother and daughter emotions and their associations with internalizing symptoms. However, all temporal processes would have been obscured. For these reasons, with these data, we believe the more traditional approaches would not have allowed us to look at socialization as conceptualized as unfolding through the mutual regulation and expression of emotion. Taken together, our findings highlight interdyad variation in patterns of emotional exchanges in different emotional contexts and suggest that the patterns that manifest during emotional exchanges may play a role in adolescents' psychosocial adjustment. These findings show how a process-oriented approach can be used to describe the bidirectional flow of emotional reactions in parent-adolescent interactions.

We found that interdyad differences in patterns of emotional exchanges mostly involved a 
subset of possible dyadic emotional states, all reflecting some combination of neutral and positive expressions (M, O, C, and E states in Figure 1). We were surprised that states involving negative emotion expressions (e.g., blue and orange cells of Figure 1) were not features of subsequences that distinguished interdyad differences in the negative emotion contexts, especially because dyads did, as a sample, show increased internalizing and externalizing expressions during the Worried/Sad and Frustrated/Annoyed discussions (see Lougheed \& Hollenstein, 2016). Instead, we found that interdyad differences largely pertained to the regulation of positive expressions: Were mothers or daughters regulating their own expression of positive emotions, and who was leading transitions to mutually-positive states? This study extends prior work examining who drives emotional content in parent-adolescent interactions (Main et al., 2016). Our results add that there are interdyad differences in whether parents or adolescents are driving transitions to positive emotions, and that these differences may be related to psychosocial adjustment.

A growing body of research is highlighting the importance of positive emotion socialization in psychosocial adjustment difficulties. For example, parents of adolescents experiencing internalizing symptoms, such as depressive symptoms, tend to dampen their adolescents' positive emotion expressions (e.g., Katz et al., 2014), and adolescents experiencing internalizing symptoms have difficulties maintaining and up-regulating positive emotion experiences (Fussner et al., 2014). Our findings add that the socialization of positive emotions, even in negative interaction contexts, may be associated with adolescent social anxiety. Specifically, during a positive interaction context (Discussion 1: Happy/Excited), mothers whose daughters showed higher social anxiety symptoms tended to express positive emotions that were not reciprocated by their daughters. Daughters experiencing higher social anxiety symptoms may 
have found it emotionally challenging to discuss positive emotions during the Emotional Rollercoaster task, or may have found the laboratory context (e.g., video cameras, physiological sensors) stressful compared to other daughters, either of which would have led to their mothers being unsuccessful in attempts to up-regulate dyadic positive emotions. Moreover, daughters experiencing lower social anxiety symptoms tended to lead the expression of positive emotions in the Worried/Sad discussion, which suggests that higher symptoms made it difficult for daughters to buffer the negative context with expressions of positive emotions. Future work could record interactions in the home environment and compare them to laboratory-based observations to more directly examine the role of adolescent social anxiety in parent-adolescent interactions.

We were surprised that we did not observe several associations that we had expected. First, we were surprised that interdyad differences in emotion patterns were not significantly associated with maternal internalizing symptoms in any of the discussions. In our previous work with this sample, mothers' symptoms, and not daughters', were primarily associated with interdyad differences in socioemotional flexibility (Lougheed \& Hollenstein, 2016). Second, we were surprised that interdyad differences in emotion patterns in the final three discussions of the Emotional Rollercoaster task (Proud, Frustrated/Annoyed, and Grateful) were not associated with mothers' or daughters' internalizing symptoms. It is impossible to determine if these null results indicate a true lack of effect, fatigue effects, or are the result of a Type II error. We speculate that a broad range of interdyad differences was not represented in our typicallydeveloping sample. We also speculate that a more diverse sample from a clinical population may show emotion patterns associated with maternal internalizing symptoms, and that interdyad differences during Proud, Frustrated/Annoyed, and Grateful discussions may be associated with 
both mothers' and daughters' symptoms.

The current study builds on prior research on emotion processes in parent-adolescent interactions by considering the entire sequence of observed dyadic emotions during interactions, in line with the assertion by Eisenberg et al. (1998) and others (e.g., Morris et al., 2007), that emotion socialization is a bidirectional process and involves the qualities of entire interactions as they unfold. Recent studies examining parent-adolescent emotion dynamics have used a topdown approach to examine dynamics of theoretical interest, determined a priori, such as synchronous or reciprocated negative emotion expressions (Moed et al., 2015; Schwartz et al., 2011; van Bommel et al., in press), and validating or supportive responses to emotions (Lougheed, Craig, et al., 2016; Lougheed, Hollenstein, et al., 2016; Main et al., 2016). Building on this work, we obtained information on emotional exchanges across entire interaction sequences and used a bottom-up, data-driven approach to examine salient patterns. The primary finding using our data-driven approach highlighted that, while dyads did express negative emotions during some emotion contexts, negative expressions did not play a role in the patterns that most distinguished interdyad differences (at least in our typically-developing sample). Rather, we found interdyad differences in whether mothers or daughters tended to drive transitions to positive expressions. Both top-down and bottom-up approaches yield valuable information on emotion dynamics and the uptake of both types of approaches will be a boon for research on emotion socialization dynamics.

\section{Limitations and Future Directions}

The results of the current study should be considered in light of several limitations. The order of the discussions in the Emotional Rollercoaster task was fixed, with all dyads experiencing the discussions in the same order. Although this aspect of the study design 
contributed to a greater standardization of procedures across participants, it was not possible to examine the extent to which transitioning between emotion contexts was associated with dyadic patterns. Future research could counterbalance the order of discussions in the Emotional Rollercoaster task to directly test how specific contextual changes are associated with dyadic dynamics in each context. In addition, the sample of mother-daughter dyads was homogenous in several respects: Only female participants were enrolled in the study, the range of adolescent age was restricted, the majority of the sample identified as European-Canadian, and both socioeconomic status and the average level of self-reported relationship quality were relatively high (see Lougheed \& Hollenstein, 2016). Thus, the results of this study might not generalize to other populations. In addition, emotional expressions and socialization vary by sex (Collins \& Russell, 1991), and it is important to examine other gender combinations of parent-adolescent dyads such as father-daughter, father-son, and mother-son. It is important to examine interdyad differences in parent-adolescent emotion socialization in samples containing more variation in internalizing symptoms and/or comparisons of dynamics in typically-developing dyads to dyads in which one or both partners are experiencing clinically-significant internalizing symptoms. Doing so is a crucial next step for examining how interdyad differences in emotion patterns are related to psychosocial adjustment outcomes.

We used grid-sequence analysis to examine emotion socialization as an indirect process (Eisenberg, Cumberland, et al., 1998) in terms of multi-step sequences of mothers' and daughters' emotion expressions. We did not capture direct socialization processes, as these data were not part of the behavioral coding system used in this study. Using grid-sequence analysis with data that captures direct socialization behaviors such as coaching, invalidation, and parental regulation of children's emotion (e.g., Eisenberg, Spinrad, et al., 1998; Morris et al., 2011) may 
allow researchers to simultaneously examine both the antecedents and consequents of direct emotion socialization. This approach will also be useful to examine socialization dynamics of other aspects of psychosocial adjustment such as externalizing symptoms, which were also not examined in the current study. Other characteristics of parent-adolescent relationships that may be important to examine in future work, in line with Eisenberg et al.'s (1998) model, include the quality of the relationship and degree of emotional closeness, which are likely play an important role in the family emotional climate.

There are a few limitations to note regarding grid-sequence analysis. First, the method assumes that each emotional expression (i.e., positive, interest, neutral, internalizing, and externalizing expressions) is equivalent in terms of its meaning and impact on the partner. Behavioral codes indicating emotional intensity and semantic content (not available in the current data) would be necessary to gain a more nuanced understanding of the social signals of emotion expressions for each dyad. Second, as with other approaches to sequence data (e.g., sequential analysis), grid-sequence analysis assumes that the temporal ordering of behaviors is meaningful in the sense that behaviors that directly precede or follow others do so in a meaningful way. As with all such methods, it is difficult or perhaps even impossible to determine if current emotional expressions are in response to a partner's most recent expression, or an expression that was made at some earlier point in the sequence. Another limitation of the method is that it may be difficult to apply to data with a high degree of missingness (Brinberg et al., 2017), which can be common in some observational data (e.g., observations with young children) but was not the case in our sample.

Grid-sequence analysis provides opportunities for several future research directions on emotion socialization dynamics. Previous work has identified age-related differences in whether 
parents or adolescents drive specific types of emotion dynamics (Main et al., 2016). It would therefore be fruitful to use grid-sequence analysis to examine changes in emotion socialization dynamics at multiple time scales (e.g., Ram \& Diehl, 2015) such as with data consisting of repeated observations of parent-adolescent interactions over months or years. Such studies would give insights into how multi-step sequences of dyadic emotion expressions develop over age or other markers of development such as puberty and would allow the examination of age-related changes in complex interpersonal dynamics. Doing so could help disentangle developmentrelated changes from interdyad differences in who tends to drive emotion dynamics in parentadolescent interactions (e.g., who tends to lead and/or repair negative emotional exchanges, who tends to up- and down-regulate negative and positive emotional expressions). Using gridsequence analysis in a data-driven way is a powerful method for reducing complex information and identifying salient patterns.

\section{Conclusion}

To echo Eisenberg et al.'s (Eisenberg, Cumberland, et al., 1998) assertions, emotion socialization is a complex process consisting of behaviors unfolding in the context of parent characteristics, child characteristics, and emotional context. It is an exciting time for research on emotion socialization as innovative quantitative analytic approaches that enable detailed examination of dynamic process are becoming more accessible. Grid-sequence analysis is one such approach that may be useful for distilling the complexity of parent-child emotion socialization dynamics. "Zooming in" on the process of emotion socialization as it unfolds during interactions allowed us to see that mothers' or daughters' tendency to regulate positive emotion dynamics across a range of interpersonal emotion contexts seems to be a distinguishing feature of typically-developing mother-daughter dyads in adolescence. 


\section{References}

Amole, M. C., Cyranowski, J. M., Wright, A. G. C., \& Swartz, H. A. (2017). Depression impacts the physiological responsiveness of mother-daughter dyads during social interaction. Depression and Anxiety, 34(2), 118-126. https://doi.org/10.1002/da.22595

Beck, A. T., Epstein, N., Brown, G., \& Steer, R. A. (1988). An inventory for measuring clinical anxiety: Psychometric properties. Journal of Consulting and Clinical Psychology, 56(6), 893-897.

Beck, A. T., Steer, R. A., \& Brown, G. K. (1996). Beck depression inventory manual (2nd ed.). San Antonio, TX: Psychological Corporation.

Brebner, J. (2003). Gender and emotions. Personality and Individual Differences, 34(3), 387394. https://doi.org/10.1016/S0191-8869(02)00059-4

Brinberg, M., Fosco, G. M., \& Ram, N. (2017). Examining inter-family differences in intrafamily (parent-adolescent) dynamics using grid-sequence analysis. Journal of Family Psychology, 31(8), 994-1004. https://doi.org/10.1037/fam0000371

Brinberg, M., Ram, N., Hülür, G., Brick, T. R., \& Gerstorf, D. (2018). Analyzing dyadic data using grid-sequence analysis: Interdyad differences in intradyad dynamics. The Journals of Gerontology Series B: Psychological Sciences and Social Sciences, 73(1), 5-18. https://doi.org/10.1093/geronb/gbw160

Collins, W. A., \& Laursen, B. (2004). Changing relationships, changing youth. The Journal of Early Adolescence, 24(1), 55-62. https://doi.org/10.1177/0272431603260882

Collins, W. A., \& Russell, G. (1991). Mother-child and father-child relationships in middle childhood and adolescence: A developmental analysis. Developmental Review, 11(2), 99136. https://doi.org/10.1016/0273-2297(91)90004-8 
Dahl, R. E. (2001). Affect regulation, brain development, and behavioral/emotional health in adolescence. CNS Spectrums, 6(1), 60-72.

de Leeuw, J., \& Mair, P. (2009). Multidimensional scaling using majorization: SMACOF in R. Journal of Statistical Software, 31(3), 1-30. https://doi.org/10.18637/jss.v031.i03

Eisenberg, N., Cumberland, A., \& Spinrad, T. L. (1998). Parental socialization of emotion. Psychological Inquiry, 9(4), 241-273. https://doi.org/10.1207/s15327965pli0904_1

Eisenberg, N., Spinrad, T. L., \& Cumberland, A. (1998). The socialization of emotion: Reply to commentaries. Psychological Inquiry, 9(4), 317-333.

Fabes, R. A., Leonard, S. A., Kupanoff, K., \& Martin, C. L. (2001). Parental coping with children's negative emotions: Relations with children's emotional and social responding. Child Development, 72(3), 907-920.

Fussner, L. M., Luebbe, A. M., \& Bell, D. J. (2014). Dynamics of positive emotion regulation: Associations with youth depressive symptoms. Journal of Abnormal Child Psychology, 43(3), 475-488. https://doi.org/10.1007/s10802-014-9916-3

Gabadinho, A., Ritschard, G., Müller, N. S., \& Studer, M. (2011). Analyzing and visualizing state sequences in R with TraMineR. Journal of Statistical Software, 40(04). https://doi.org/10.18637/jss.v040.i04

Gottman, J. M., McCoy, K., Coan, J., \& Collier, H. (1995). The Specific Affect Coding System (SPAFF) for observing emotional communication in marital and family interaction. Mahwah, NJ: Erlbaum.

Graber, J. A., Sontag, L. M., Lerner, R. M., \& Steinberg, L. (2004). Internalizing problems during adolescence. In Handbook of adolescent psychology (pp. 587-626). John Wiley \& Sons. 
Granic, I., \& Lougheed, J. P. (2016). The role of anxiety in coercive family processes with aggressive children. In T. J. Dishion \& J. Snyder (Eds.), The Oxford Handbook of Coercive Relationship Dynamics (pp. 231-248). Oxford, England: Oxford University Press.

Hastings, P. D., Klimes-Dougan, B., Kendziora, K. T., Brand, A., \& Zahn-Waxler, C. (2014). Regulating sadness and fear from outside and within: Mothers' emotion socialization and adolescents' parasympathetic regulation predict the development of internalizing difficulties. Development and Psychopathology, 26, 1369-1384. https://doi.org/10.1017/S0954579414001084

Hollenstein, T. (2013). State space grids: Depicting dynamics across development. New York: Springer.

Hout, M. C., Papesh, M. H., \& Goldinger, S. D. (2013). Multidimensional scaling. Wiley Interdisciplinary Reviews: Cognitive Science, 4(1), 93-103. https://doi.org/10.1002/wcs.1203

Katz, L. F., Shortt, J. W., Allen, N. B., Davis, B., Hunter, E., Leve, C., \& Sheeber, L. B. (2014). Parental emotion socialization in clinically depressed adolescents: Enhancing and dampening positive affect. Journal of Abnormal Child Psychology, 42(2), 205-215. https://doi.org/10.1007/s10802-013-9784-2

Klimes-Dougan, B., Brand, A. E., Zahn-Waxler, C., Usher, B., Hastings, P. D., Kendziora, K., \& Garside, R. B. (2007). Parental emotion socialization in adolescence: Differences in sex, age and problem status. Social Development, 16(2), 326-342. https://doi.org/10.1111/j.1467-9507.2007.00387.x

Koffer, R. E., Ram, N., \& Almeida, D. M. (2017). More than counting: An intraindividual 
variability approach to categorical repeated measures. The Journals of Gerontology:

Series B, 73(1), 87-99. https://doi.org/10.1093/geronb/gbx086

Kruskal, J. (1964). Multidimensional scaling by optimizing goodness of fit to a nonmetric hypothesis. Psychometrika, 29(1), 1-27.

Kruskal, J. B. (1983). An overview of sequence comparison: Time warps, string edits, and macromolecules. SIAM Review, 25(2), 201-237.

Liebowitz, M. R. (1987). Social phobia. Karger Publishers.

Lougheed, J. P., Craig, W. M., Pepler, D., Connolly, J., O’Hara, A., Granic, I., \& Hollenstein, T. (2016). Maternal and peer regulation of adolescent emotion: Associations with depressive symptoms. Journal of Abnormal Child Psychology, 44(5), 963-974.

https://doi.org/10.1007/s10802-015-0084-x

Lougheed, J. P., \& Hollenstein, T. (2014). Specific Affect Coding System Manual: 5-Code Version. Unpublished manual.

Lougheed, J. P., \& Hollenstein, T. (2016). Socioemotional flexibility in mother-daughter dyads: Riding the emotional rollercoaster across positive and negative contexts. Emotion, 16(5), 620-633. https://doi.org/10.1037/emo0000155

Lougheed, J. P., Hollenstein, T., \& Lewis, M. D. (2016). Maternal regulation of daughters’ emotion during conflicts from early to mid-adolescence. Journal of Research on Adolescence, 26(3), 610-616. https://doi.org/10.1111/jora.12211

Lougheed, J. P., Hollenstein, T., Lichtwarck-Aschoff, A., \& Granic, I. (2015). Maternal regulation of child affect in externalizing and typically-developing children. Journal of Family Psychology, 29(1), 10-19. https://doi.org/10.1037/a0038429

Main, A., Paxton, A., \& Dale, R. (2016). An exploratory analysis of emotion dynamics between 
mothers and adolescents during conflict discussions. Emotion, 16(6), 913-928.

https://doi.org/10.1037/emo0000180

Moed, A., Gershoff, E. T., Eisenberg, N., Hofer, C., Losoya, S., Spinrad, T. L., \& Liew, J. (2015).

Parent-adolescent conflict as sequences of reciprocal negative emotion: Links with conflict resolution and adolescents' behavior problems. Journal of Youth \& Adolescence, 44(8), 1607-1623. https://doi.org/10.1007/s10964-014-0209-5

Morgan, J. K., Ambrosia, M., Forbes, E. E., Cyranowski, J. M., Amole, M. C., Silk, J. S., ... Swartz, H. A. (2015). Maternal response to child affect: Role of maternal depression and relationship quality. Journal of Affective Disorders, 187, 106-113. https://doi.org/10.1016/j.jad.2015.07.043

Morris, A. S., Criss, M. M., Silk, J. S., \& Houltberg, B. J. (2017). The impact of parenting on emotion regulation during childhood and adolescence. Child Development Perspectives, 11(4), 233-238. https://doi.org/10.1111/cdep.12238

Morris, A. S., Cui, L., Criss, M. M., \& Simmons, W. K. (2018). Emotion regulation dynamics during parent-child interactions: Implications for research and practice. In P. M. Cole \& T. Hollenstein (Eds.), Emotion Regulation: A Matter of Time. New York: Routledge.

Morris, A. S., Silk, J. S., Morris, M. D. S., Steinberg, L., Aucoin, K. J., \& Keyes, A. W. (2011). The influence of mother-child emotion regulation strategies on children's expression of anger and sadness. Developmental Psychology, 47(1), 213-225. https://doi.org/10.1037/a0021021

Morris, A. S., Silk, J. S., Steinberg, L., Myers, S. S., \& Robinson, L. R. (2007). The role of the family context in the development of emotion regulation. Social Development, 16(2), 361-388. https://doi.org/10.1111/j.1467-9507.2007.00389.x 
Myers, M. G., Stein, M. B., \& Aarons, G. A. (2002). Cross validation of the Social Anxiety Scale for Adolescents in a high school sample. Journal of Anxiety Disorders, 16(2), 221-232.

Pitzer, L. M., \& Bergeman, C. S. (2014). Synchrony in affect among stressed adults: The Notre Dame widowhood study. The Journals of Gerontology Series B: Psychological Sciences and Social Sciences, 69B(1), 29-39. https://doi.org/10.1093/geronb/gbt026

R Core Team. (2018). R: A Language and Environment for Statistical Computing. Retrieved from https:/www.R-project.org/

Ram, N., Benson, L., Brick, T. R., Conroy, D. E., \& Pincus, A. L. (2017). Behavioral landscapes and earth mover's distance: A new approach for studying individual differences in density distributions. Journal of Research in Personality, 69, 191-205. https://doi.org/10.1016/j.jrp.2016.06.010

Ram, N., \& Diehl, M. (2015). Multiple time-scale design and analysis: Pushing towards realtime modeling of complex developmental processes. In M. Diehl, K. Hooker, \& M. J. Sliwinski (Eds.), Handbook of intraindividual variability across the lifespan (pp. 308323). New York: Routledge.

Ramsey, M. A., \& Gentzler, A. L. (2015). An upward spiral: Bidirectional associations between positive affect and positive aspects of close relationships across the life span. Developmental Review, 36, 58-104. https://doi.org/10.1016/j.dr.2015.01.003

Ritschard, G., Bürgin, R., \& Studer, M. (2013). Exploratory mining of life event histories. In J. J. McArdle \& G. Ritschard (Eds.), Contemporary Issues in Exploratory Data Mining in the Behavioral Sciences (pp. 221-253). New York: Routledge.

Schwartz, O. S., Dudgeon, P., Sheeber, L. B., Yap, M. B. H., Simmons, J. G., \& Allen, N. B. (2011). Observed maternal responses to adolescent behaviour predict the onset of major 
depression. Behaviour Research and Therapy, 49(5), 331-338.

https://doi.org/10.1016/j.brat.2011.02.008

Shannon, C. E. (1948). A mathematical theory of communication. The Bell System Technology Journal, 27, 379-423. https://doi.org/10.1002/j.1538-7305.1948.tb01338.x

Smetana, J. G., Campionne-Barr, N., \& Metzger, A. (2006). Adolescent development in interpersonal and societal contexts. Annual Review of Psychology, 57, 255-284. https://doi.org/10.1146/annurev.psych.57.102904.190124

Studer, M., \& Ritschard, G. (2016). What matters in differences between life trajectories: A comparative review of sequence dissimilarity measures. Journal of the Royal Statistical Society: Series A (Statistics in Society), 179(2), 481-511. https://doi.org/10.1111/rssa.12125

van Bommel, D. M. H., van der Giessen, D., van der Graaff, J., Meeus, W. H. J., \& Branje, S. J. T. (in press). Mother-adolescent conflict interaction sequences: The role of maternal internalizing problems. Journal of Research on Adolescence. https://doi.org/10.1111/jora.12441

van der Giessen, D., Branje, S. J. T., Frijns, T., \& Meeus, W. H. J. (2013). Dyadic variability in mother-adolescent interactions: Developmental trajectories and associations with psychosocial functioning. Journal of Youth and Adolescence, 42(1), 96-108. https://doi.org/10.1007/s10964-012-9790-7

Walle, E. A., \& Campos, J. J. (2012). Interpersonal responding to discrete emotions: A functionalist approach to the development of affect specificity. Emotion Review, 4(4), 413-422. https://doi.org/10.1177/1754073912445812

Wickham, H. (2007). Reshaping data with the reshape package. Journal of Statistical Software, 
21(12). https://doi.org/10.18637/jss.v021.i12

Wickham, H. (2009). ggplot2: Elegant Graphics for Data Analysis. Retrieved from http://ggplot2.org 
Table 1

Interdyad Differences in Emotion Patterns: Description of Sequence Dimensions

\begin{tabular}{|c|c|c|c|c|}
\hline \multirow[b]{2}{*}{ Dimension } & \multirow[b]{2}{*}{ Most Discriminating Subsequence } & \multirow[b]{2}{*}{$X^{2}$} & \multicolumn{2}{|c|}{ Proportional Frequency } \\
\hline & & & $\begin{array}{l}\text { Lowest } \\
\text { Quartile }\end{array}$ & $\begin{array}{l}\text { Highest } \\
\text { Quartile }\end{array}$ \\
\hline (1) Mother regulating mutual positive to & $\begin{array}{l}\text { Discussion } 1 \text { (Happy/Excited }) \\
(\mathrm{E}>\mathrm{C})-(\mathrm{E}>\mathrm{C})-(\mathrm{C}>\mathrm{M})-(\mathrm{M}>\mathrm{C})\end{array}$ & $32.25^{*}$ & $0.21 *$ & $1.00 *$ \\
\hline (2) Mother regulating positive & Discussion $2($ Worried/Sad $)$ & 42.14 & $0.1 J$ & 0.05 \\
\hline $\begin{array}{l}\text { (1) Daughter down-regulating mutual } \\
\text { positive }\end{array}$ & $(\mathrm{E}>\mathrm{O})$ & $19.88^{*}$ & $0.21 *$ & 0.58 \\
\hline (2) Daughter regulating positive & $(\mathrm{C}>\mathrm{M})-(\mathrm{M}>\mathrm{C})-(\mathrm{M}>\mathrm{C})$ & $27.69 *$ & $0.13 *$ & $0.88 *$ \\
\hline $\begin{array}{l}\text { (1) Daughter regulating positive to daughter } \\
\text { up-regulating mutual positive }\end{array}$ & $\begin{array}{r}\text { Discussion } 3 \text { (Proud) } \\
(\mathrm{M})-(\mathrm{M}>\mathrm{C})-(\mathrm{M}>\mathrm{C})-(\mathrm{C}>\mathrm{E})\end{array}$ & $31.69 *$ & $0.17 *$ & 0.83 \\
\hline (2) Mother regulating mutual positive & $(\mathrm{E}>\mathrm{C})-(\mathrm{E}>\mathrm{C})$ & $30.00 *$ & $0.25 *$ & $0.92 *$ \\
\hline $\begin{array}{l}\text { (1) Mother up-regulating mutual positive } \\
\text { (2) Daughter up-regulating mutual positive } \\
\text { to daughter regulating positive }\end{array}$ & $\begin{array}{l}\text { Discussion } 4 \text { (Frustrated/Annoyed) } \\
(\mathrm{M})-(\mathrm{O}>\mathrm{E}) \\
(\mathrm{M})-(\mathrm{M}>\mathrm{C})-(\mathrm{C}>\mathrm{E})-(\mathrm{C}>\mathrm{M})>(\mathrm{C}>\mathrm{M})\end{array}$ & $\begin{array}{l}25.07^{*} \\
50.73^{*}\end{array}$ & $\begin{array}{l}0.88 \\
1.00 *\end{array}$ & $\begin{array}{l}0.33^{*} \\
0.13^{*}\end{array}$ \\
\hline $\begin{array}{l}\text { (1) Mother up-regulating mutual positive to } \\
\text { mother regulating positive } \\
\text { (2) Daughter regulating positive }\end{array}$ & $\begin{array}{l}\text { Discussion } 5 \text { (Grateful) } \\
(\mathrm{M})-(\mathrm{O}>\mathrm{M})-(\mathrm{E}>\mathrm{O})-(\mathrm{O}>\mathrm{M})\end{array}$ & $32.42 *$ & $0.17 *$ & $0.92 *$ \\
\hline
\end{tabular}

Note. $* p<.05$. Transitions between dyadic states are enclosed in parentheses and indicated by $>$. Hyphens (-) indicate a time gap in which a state is remained in for some duration of time. 
Table 2

Results for Linear Regressions Examining Relation Between Symptoms and Interdyad Differences in Mother-Daughter Sequences in Discussion 1 (Happy/Excited) and Discussion 2 (Worried/Sad)

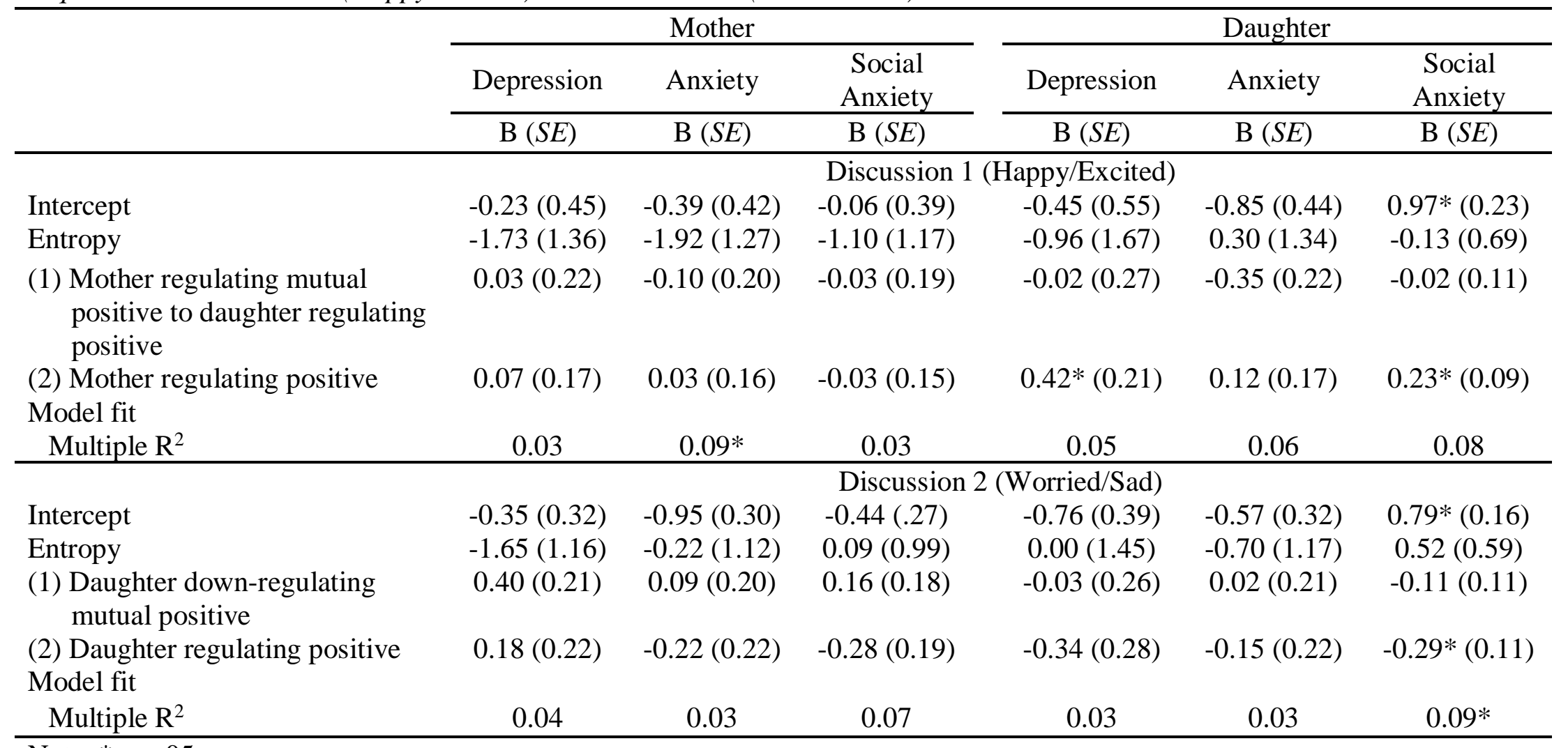

Note. ${ }^{*} p<.05$. 
Table 3

Results for Linear Regressions Examining Relation between Symptoms and Interdyad Differences in from Mother-Daughter Sequences in Discussion 3 (Proud), Discussion 4 (Frustrated/Annoyed), and Discussion 5 (Grateful)

\begin{tabular}{|c|c|c|c|c|c|c|}
\hline & \multicolumn{3}{|c|}{ Mother } & \multicolumn{3}{|c|}{ Daughter } \\
\hline & Depression & Anxiety & $\begin{array}{l}\text { Social } \\
\text { Anxiety }\end{array}$ & Depression & Anxiety & $\begin{array}{l}\text { Social } \\
\text { Anxiety }\end{array}$ \\
\hline & $\mathrm{B}(S E)$ & $\mathrm{B}(S E)$ & $\mathrm{B}(S E)$ & $\mathrm{B}(S E)$ & $\mathrm{B}(S E)$ & $\mathrm{B}(S E)$ \\
\hline & \multicolumn{6}{|c|}{ Discussion 3 (Proud) } \\
\hline Intercept & $-0.40(0.42)$ & $-0.93 *(0.39)$ & $-0.10(0.35)$ & $-0.68(0.51)$ & $-1.01 *(0.41)$ & $0.96 *(0.21)$ \\
\hline Entropy & $-1.49(1.56)$ & $-0.31(1.44)$ & $-1.17(1.30)$ & $-0.30(1.92)$ & $0.98(1.54)$ & $-0.12(0.78)$ \\
\hline $\begin{array}{l}\text { (1) Daughter regulating positive to daughter } \\
\text { up-regulating mutual positive }\end{array}$ & $0.02(0.29)$ & $-0.29(0.26)$ & $-0.10(0.24)$ & $0.12(0.35)$ & $-0.33(0.28)$ & $0.10(0.14)$ \\
\hline (2) Mother regulating mutual positive & $0.02(0.17)$ & $-0.21(0.16)$ & $0.18(0.15)$ & $-0.26(0.21)$ & $-0.26(0.17)$ & $-0.16(0.09)$ \\
\hline \multicolumn{7}{|l|}{ Model fit } \\
\hline Multiple $\mathrm{R}^{2}$ & 0.03 & $0.08 *$ & 0.07 & 0.03 & 0.04 & 0.06 \\
\hline & \multicolumn{6}{|c|}{ Discussion 4 (Frustrated/Annoyed) } \\
\hline Intercept & $-0.58(0.36)$ & $-1.05 *(0.35)$ & $-0.43(0.31)$ & $-0.83(0.46)$ & $-0.41(0.36)$ & $0.92 *(0.19)$ \\
\hline Entropy & $-0.68(1.11)$ & $0.11(1.07)$ & $0.06(0.96)$ & $0.22(1.41)$ & $-1.07(1.12)$ & $0.01(0.59)$ \\
\hline (1) Mother up-regulating mutual positive & $-0.27(0.25)$ & $0.34(0.24)$ & $-0.32(0.21)$ & $0.08(0.32)$ & $-0.01(0.25)$ & $0.01(0.13)$ \\
\hline $\begin{array}{l}\text { (2) Daughter up-regulating mutual positive } \\
\text { to daughter regulating positive }\end{array}$ & $0.10(0.15)$ & $0.17(0.14)$ & $-0.03(0.13)$ & $0.24(0.19)$ & $0.25(0.15)$ & $0.08(0.08)$ \\
\hline \multicolumn{7}{|l|}{ Model fit } \\
\hline Multiple $\mathrm{R}^{2}$ & 0.08 & 0.07 & 0.06 & 0.02 & 0.05 & 0.01 \\
\hline & \multicolumn{6}{|c|}{ Discussion 5 (Grateful) } \\
\hline Intercept & $-0.81 *(0.37)$ & $-1.18 *(0.35)$ & $-0.22(0.31)$ & $-0.31(0.45)$ & $-0.67(0.37)$ & $0.88 *(0.19)$ \\
\hline Entropy & $0.08(1.45)$ & $0.66(1.37)$ & $-0.77(1.25)$ & $-1.82(1.80)$ & $-0.34(1.47)$ & $0.17(0.76)$ \\
\hline $\begin{array}{l}\text { (1) Mother up-regulating mutual positive to } \\
\text { mother regulating positive }\end{array}$ & $-0.18(0.28)$ & $-0.37(0.27)$ & $-0.04(0.24)$ & $0.44(0.35)$ & $0.06(0.29)$ & $0.04(0.15)$ \\
\hline (2) Daughter regulating positive & $-0.18(0.19)$ & $-0.23(0.18)$ & $0.00(0.16)$ & $0.04(0.23)$ & $-0.11(0.19)$ & $-0.01(0.10)$ \\
\hline \multicolumn{7}{|l|}{ Model fit } \\
\hline Multiple $\mathrm{R}^{2}$ & 0.02 & 0.05 & 0.02 & 0.02 & 0.01 & 0.01 \\
\hline
\end{tabular}

Note. ${ }^{*} p<.05$. 
A

B
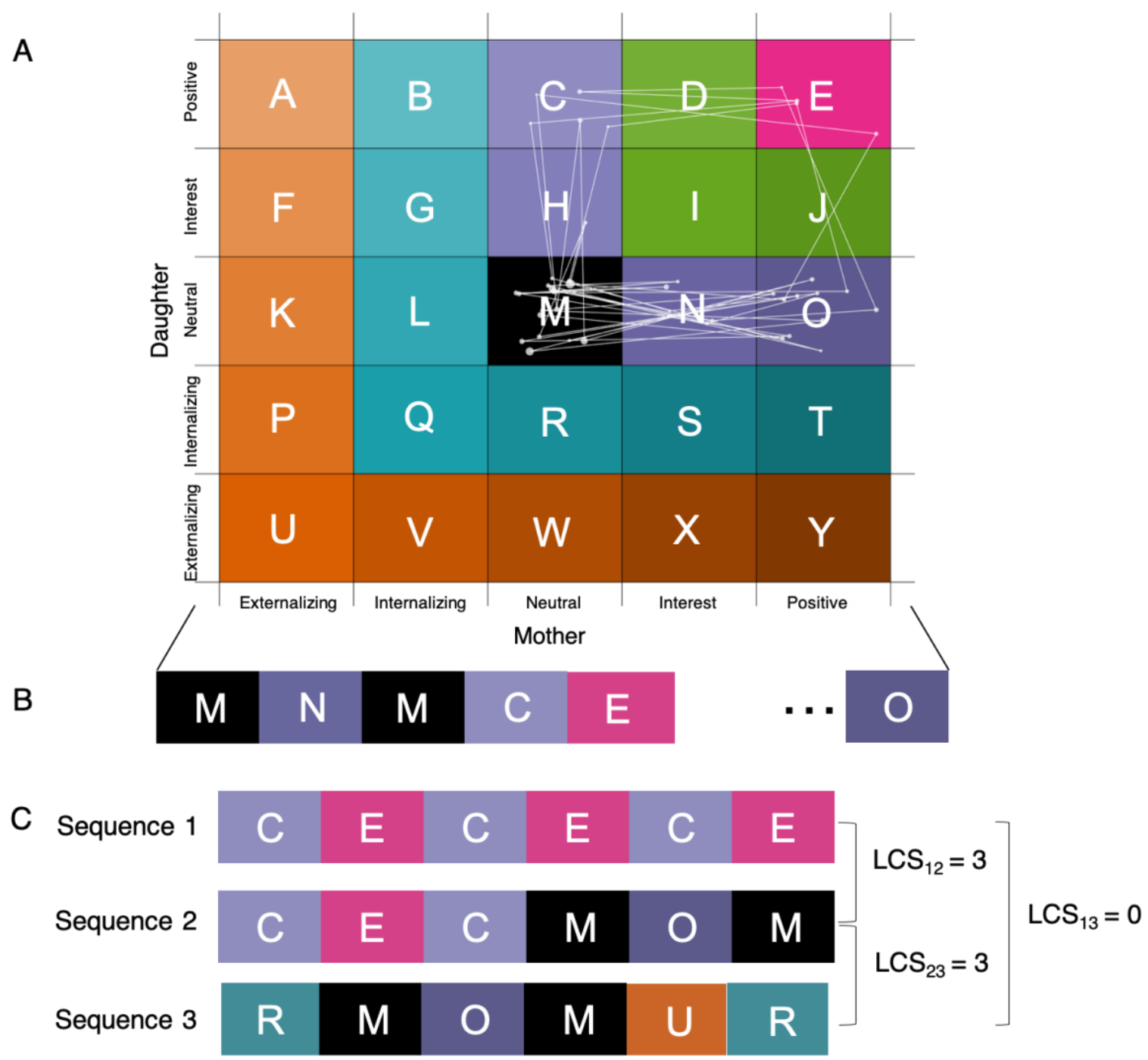

Figure 1. Steps 2 and 3 of grid-sequence analysis. Panel A shows a state space grid for one dyad in Discussion 1 (Happy/ Excited). Panel B shows the categorical dyadic time series extracted from the state space grid for one dyad. Panel $\mathrm{C}$ shows the calculation of the length of the longest common subsequence used in calculating the dissimilarity among three hypothetical dyads' sequences. 